\title{
Registration, Orientation and Edge Detection from Digital Mammogram Images
}

\author{
Indra Kanta Maitra ${ }^{1}$ and Samir Kumar Bandyopadhyay ${ }^{2}$ \\ ${ }^{1}$ B.P. PIM\&T, Kolkata, West Bengal, 700052, India \\ ${ }^{2}$ Advisor to Chancellor, JIS University, Kolkata, West Bengal 700109, India
}

\begin{abstract}
Computer-Aided Diagnosis (CAD) systems require computational algorithms on medical images for the detection of breast cancer. This breast cancer is causing to women is the serious concern and serious diseases that were increasing day to day in death rates for women. This paper focused on the Registration, Orientation and edge detection from digital mammogram images utilizing image processing. The proposed method has been tested on all mammograms from standard mammogram database, and extensive quantitative analysis with the classical methods is done.
\end{abstract}

Keywords: DCHEA, MDT and EDA, Registration, Views of breast

\section{Introduction}

Mammogram registration and edge detection play a critical step in the automatic detection of breast anomalies. In this paper, a new hybrid mammogram registration method has been proposed combining anatomical features and intensity-based homogeneity features for image registration techniques. The output of the preparation process is the input of the proposed algorithms. To enhance the homogeneity of the image, a new algorithm is proposed by me, namely, Divide and Conquer Homogeneity Enhancement Algorithm (DCHEA). It not only homogenizes the image but also quantized colour up to the application level in grey-scale. Here five alternative methods, i.e. First Value, Min-Max, Mean, Median and Mode, are applied to obtain the central tendencies of image intensities to propagate, so that, to obtain the homogenous image. The former includes skin boundary [1] and the nipple [2], while the latter includes the pectoral muscle, fibro-glandular tissue, and vasculature. The homogeneity process is followed by the detection of edges in an image.

\section{Literature review}

Over the past 20 years, continuous advancement in the field of image processing algorithms, computer technology in terms of computational power and storage, and digital medical imaging technologies have allowed the development of powerful computer-assisted analytical tools towards a diagnosis of critical diseases like cancer [3][4]. Last few years, several states of the art Computer-Aided Diagnosis (CAD) systems have been developed to deal with different fields of disease analysis. The breast cancer diagnosis is one of the most

Article history:

Received (December 23, 2019), Review Result (February 3, 2020), Accepted (March 8, 2020) 
well-accepted implementations of CAD to assist medical practitioners [5][6][7]. Breast cancer identification by image processing also follows the same path.

\section{Proposed methodology}

Very few mammographic datasets are available in the public domain for academic research activity. Among the three mammographic databases discussed above, MIAS dataset is sufficiently large to conduct experimental analysis. Moreover, the dataset contains 322 mammogram images of different size, shape and morphology. The images are also classified and benchmarked by an expert team of radiologist of MIAS into three distinct categories based on their parenchymal density.

Further, the radiologist has provided all relevant information regarding any abnormality present. The images are classified into normal, asymmetry, presence of mass, calcification etc. The mass is further classified according to the type of mass present. The choice of MIAS database for conducting most of the experiments described in this paper is due to its sufficiently large volume, image quality, easy availability and several benchmarked cases. Most of the critical international research work in this area is conducted using MIAS dataset as evident in most of the dissertations. So MIAS dataset has been used to prove the efficiency of my proposed methods, quantitative assessment and to compare the results with other vital dissertations.

To achieve the desired goal, a new algorithm has been introduced by the combination of modified seeded region growing with thresholding.

\subsection{Algorithm: Proposed seeded region growing artefact removal algorithm}

SEEDED-REGION-GROWING (OrgImage, ImgWidth, ImgHeight)

$\Delta t \leftarrow 10$

If OrgImage[ImgHeight/2, ImgWidth*3/4]. Intensity $>\Delta t$

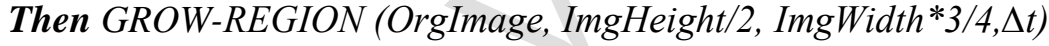

Else If OrgImage[ImgHeight/2, ImgWidth*5/8]. Intensity $>\Delta t$

Then GROW-REGION (OrgImage, ImgHeight/2, ImgWidth*5/8, $\Delta t$ )

Else If OrgImage[ImgHeight/2, ImgWidth/2]. Intensity $>\Delta t$

Then GROW-REGION (OrgImage, ImgHeight/2, ImgWidth/2, $\Delta t$ )

Else Error

Return

GROW-REGION (OrgImage, $h, w, \Delta t)$ Stack $\leftarrow$ New Empty Stack NewImage $\leftarrow$ New Blank Image Stack.Push (h)

Stack.Push (w)

While Stack $\neq$ Empty

Do

$x \leftarrow$ stack.Pop ()

$y \leftarrow$ stack.Pop ()

GreyValue $\leftarrow$ OrgImage [x,y].Intensity

If GreyValue $>\Delta t$

Then NewImage $[x, y]$.Intensity $\longleftarrow$ GreyValue

OrgImage $[x, y] . I n t e n s i t y ~ \longleftarrow 0$

If $x-1>0$ AND OrgImage $[x-1, y]$.Intensity $>\Delta t$

Then Stack.Push (y)

Stack.Push $(x-1)$ 
If $x+1<$ OrgImage.height AND OrgImage $[x+1, y]$.Intensity $>\Delta t$

Then Stack.Push (y)

Stack.Push $(x+1)$

If $y-1>0$ AND OrgImage $[x, y$-1].Intensity $>\Delta t$

Then Stack.Push (y-1)

Stack.Push $(x)$

If $y+1<$ OrgImage.width AND OrgImage $[x, y+1]$.Intensity $>\Delta t$

Then Stack.Push $(y+1)$ Stack.Push (x)

Return (NewImage)

[Figure 1] shows Original Mammogram and Mammogram without external artefact after the operation of the proposed algorithm.

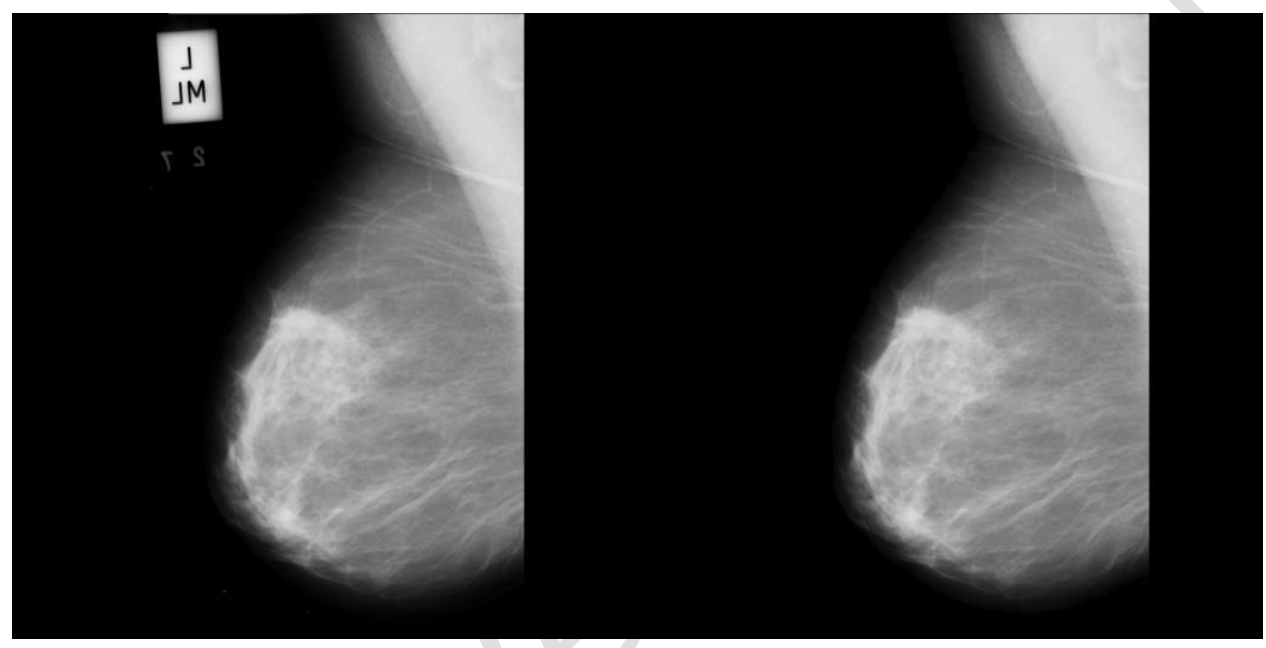

Figure 1. Original mammogram and mammogram without external artefact after SRGA

One of the important conditions of a fully automated system is to standardize the input. So at this phase, it is needed to identify the entire breast mammograms with a left orientation for the execution of developed algorithms.

\subsection{Algorithm: Homogeneous orientation using image orientation algorithm}

HOMO-ORIENTATION (OrgImage, Height, Width) ChestWall $\longleftarrow 0$

$\Delta d \leftarrow$ Constant

Then Loop I ᄂO to Height

Do $\quad$ Loop $j \longleftarrow 0$ toWidth

Do If OrgImage $[i, j]>0$

Then If $i=0$

Then ChestWall $\leftarrow j$

If ChestWall $\neq j$

Then HORZ-FLIP (OrgImage, Height, Width)

Return

HORZ-FLIP (OrgImage, Width, Height) NewImage $\longleftarrow$ New Blank Image Loop $i \longleftarrow 0$ toHeight

Do

Loop $j \longleftarrow 0$ to Width

Do NewImage $[i, j]$. Intensity $\leftarrow$ OrgImage $[i$, Width $-(j+1)]$. Intensity 


\section{Return (NewImage)}

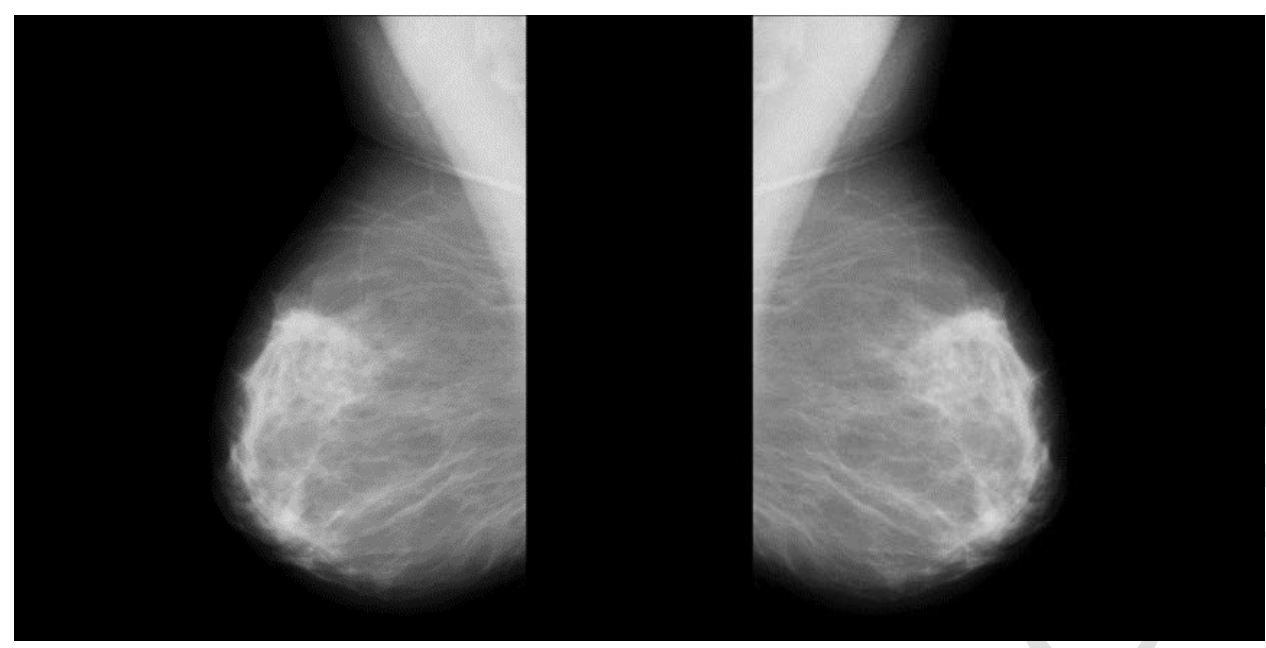

Figure 2. Right mammogram and mammogram after flipping $180^{\circ}$

Mammogram registration and edge detection play a critical step in the automatic detection of breast anomalies. The proposed Histogram with equalization had been given in detail below and discussed.

\subsection{Algorithm: Proposed histogram equalization}

LEVEL-HISTOGRAM (Histogram, NumberBin) $n$ NumberBin

Loop $x \leftarrow 1$ ton

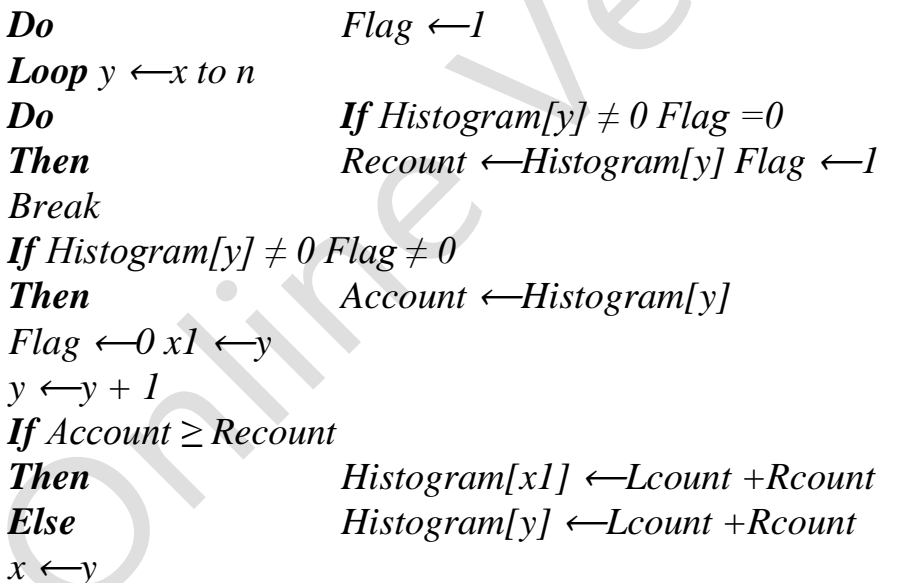

\section{Return Histogram}

The Average Bin Distance (ABD) is the mean of different bin distance in the Histogram.

\subsection{Algorithm: Calculation of average bin distance}

BIN-DISTANCE (Histogram, NumberBin) $n \leftarrow$ NumberBin

SumDistance $\longleftarrow 0$

Count $\longleftarrow 0$

HistBin $\longleftarrow 0$

Loop $\quad x \leftarrow 1$ ton 


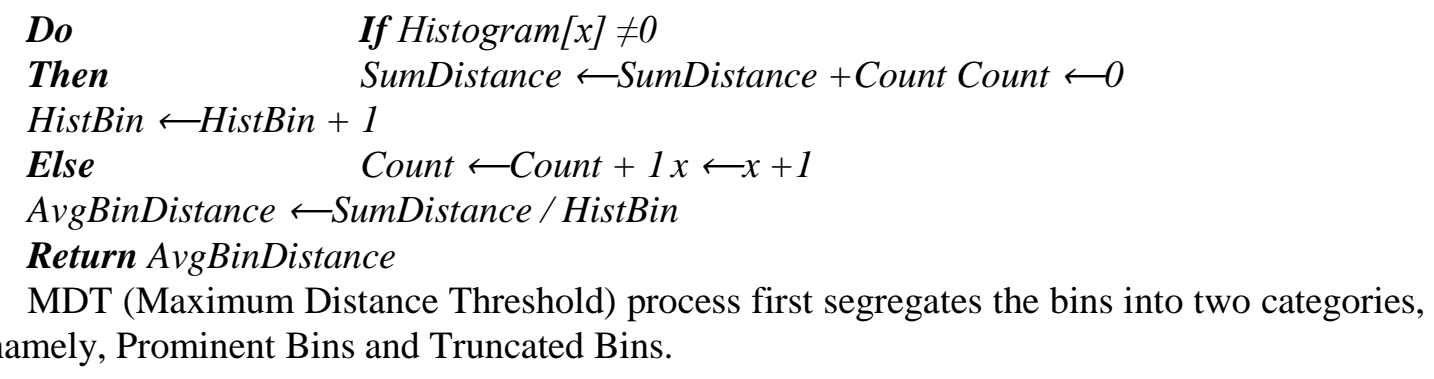

\subsection{Algorithm: Calculation of mdt by identifying the prominent bins}

CALCULATE-MDT (Histogram, NumberBin, AvgBinDistance) $n \leftarrow$ NumberBin

TotPrmBinDistance $\longleftarrow 0$

Count $\longleftarrow 0$

TotPrmBin $\longleftarrow 0$

Loop $\quad x \leftarrow 1$ ton

Do If Histogram $[x] \neq 0$

Then If Count $\geq A v g$ BinDistance

ThenTotPrmBinDistance $\leftarrow$ TotPrmBinDistance + Count

TotPrmBin $\leftarrow$ TotPrmBin +1 Count $\longleftarrow 0$

Else Count $\longleftarrow+1$

Else Count $\leftarrow$ Count $+1 x \leftarrow x+1$

MDT $\leftarrow$ TotPrmBinDistance / TotPrmBin

Return MDT

The algorithm is divided into two parts. According to this method, a Maximum Difference Threshold (MDT) value has been already calculated, which a constant threshold is. In the first part, the proposed method traverses the image in row-major order. The algorithm scans the image pixel horizontally from left to right and from top to bottom. The algorithm calculates subtraction between two subsequent pixels if the absolute value of the result is greater than MDT the row will be divided into two subsets along with the point of difference. In the second part, the same process will be repeated column-major order.

\subsection{Algorithm: Divide and conquer homogeneity enhancement}

DivConquerHomgEnht (OrgImage, Height, Width, MDT)

\section{Loop $x \leftarrow 1$ to Height}

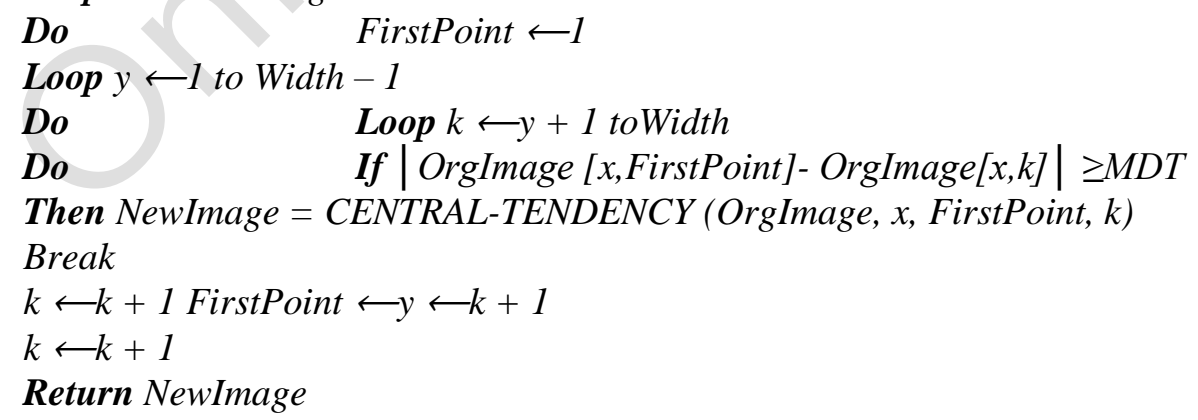




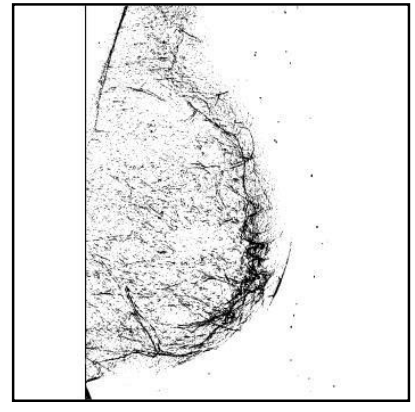

(a)

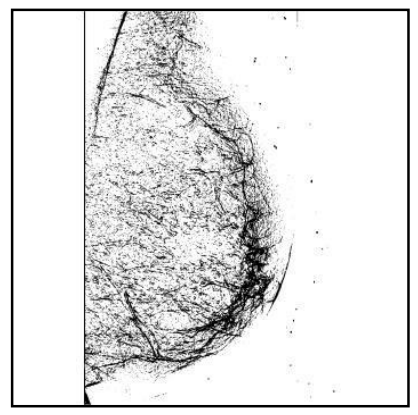

(d)

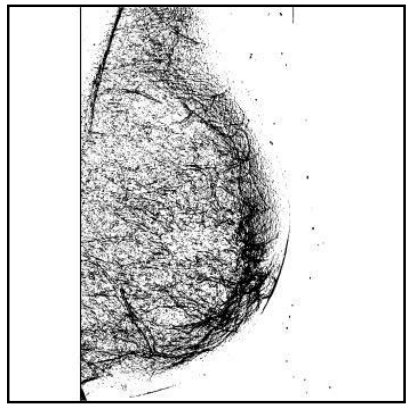

(b)

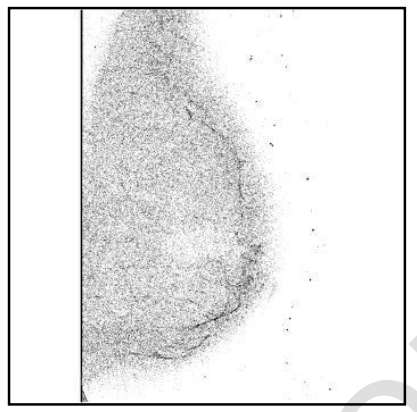

(e)

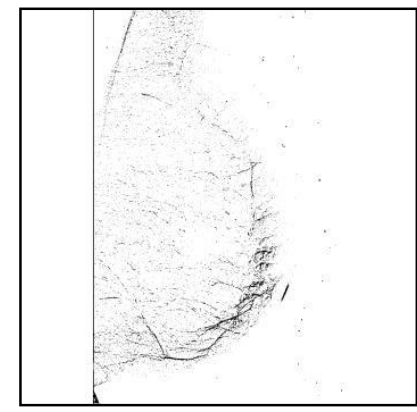

(c)

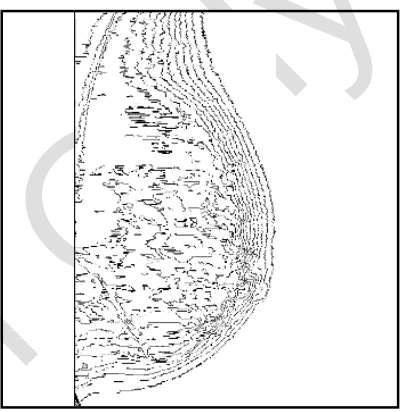

(f)

Figure 3. Detected edge of mammogram image (mdb014) with (a) Roberts' cross, (b) Sobel filter, (c) Prewitt filter, (d) Kirsch operator, (e) Laplacian of Gaussian (LoG) and (f) Proposed EDA edge map

The output of DCHEA with mode value as the central tendency measure is the input image of the proposed edge detection algorithm. The choice of statistical Mode value over others is due to its better edge detection capacity as described in the quantitative analysis section of this chapter. Furthermore, instead of using conventional edge detection algorithms that use a convolution filter, a new alternative method has been proposed. The proposed method has been tested on all mammograms from standard mammogram database, and analysis with the classical methods is done. The edge map image is free from any noise and is visible.

\section{Conclusions}

Preparation processing has been performed on all the images before the execution of the algorithms. DCHEA has obtained a homogenous image with colour quantized to the desired level of grey-scales by using MDT. EDA edge detection has been performed on the homogenous image. The proposed method has been tested on all mammograms from standard mammogram database, and extensive quantitative analysis with the classical methods is done.

\section{References}

[1] P. Hogg, J. Kelly, and C. Mercer, "Digital mammography: A holistic approach", Springer, (2017)

[2] Hopp T and Ruitter NV, "2D/3D Registration for localization of mammographically depicted lesions in breast MRI,” Lecture Notes in Computer Science, vol.7361, pp.627-634, (2012)

[3] H. John, V. Vasileios, H. Lianghao, M. Thomy, E. Bjoern, and H. David, "A review of biomechanically informed breast image registration," Physics in Medicine and Biology, vol.61, no.2, (2016)

[4] D. Lo, "Finite element mesh generation," Boca Raton, FL: CRC Press, (2015) 
[5] A. Siva Pavan, Ch.Sudhakar, S. Naga Mallik Raj, "Problems in digital image processing: A survey," International Journal of Image and Signal Systems Engineering, vol.3, no.1, pp.7-14, (2019) DOI:10.21742/IJISSE.2019.3.1.02

[6] S. Juan, R. Maria, C. Monteserrat, F. Eric, G. Mila, and L. Maximiliano, "A complete software application for automatic registration of X-ray mammography and magnetic resonance images," Physics in Medicine and Biology, (2014) DOI:10.1118/1.4885957

[7] Kai Zhang, N.Thirupathi Rao, Debnath Bhattacharyya, "Matrix depiction based cyst detection in pediatric aged MRI/ ULTRASONIC and ultrasonic images," International Journal of Smart Home, vol.12, no.4, pp.2738, (2018) DOI:10.21742/IJSH.2018.12.4.04 
Registration, Orientation and Edge Detection from Digital Mammogram Images

This page is empty by intention. 